\title{
Is hormone therapy during climacteric for all?
}

\section{TERAPIA HORMONAL NO CLIMATÉRIO É PARA TODAS?}

\author{
José Maria Soares Júnior ${ }^{1}$, Isabel C. Espósito Sorpreso², Edmund C. Baracat ${ }^{3}$ \\ ${ }^{1}$ Associate professor of Gynecology in the Department of Obstetrics and Gynecology, Hospital das Clínicas, Faculdade de Medicina da Universidade de São Paulo (HC-FMUSP), São Paulo, SP, Brazil \\ ${ }^{2}$ Associate professor of Gynecology in the Department of Obstetrics and Gynecology, HC-FMUSP, São Paulo, SP, Brazil \\ ${ }^{3}$ Full professor of Gynecology in the Department of Obstetrics and Gynecology, HC-FMUSP, São Paulo, SP
}

Climacteric is a period of changes in women. It is the transition from childbearing years to a non-reproductive stage. It is surrounded by myths and fears. Etymologically, it means critical time; it has, therefore, a negative connotation. Many women do not experience symptoms or major difficulties in this transition phase. But there is a group in which the effect of estrogen deprivation is severe to the point of impacting the quality of life and interpersonal, family and professional relationships. ${ }^{1}$ In general, these women complain of severe vasomotor symptoms (hot flashes), which may include sweating and disrupt sleep when they happen overnight. ${ }^{2}$ For them, the best treatment is that with estrogen therapy, but there are still restrictions on its use. ${ }^{3}$

The debate over the use of hormone therapy in menopause is old, from the 1963 feminist movement "Feminine Forever" to the first reports of cases of endometrial and breast cancer, culminating with the apocalyptic study Women Health Initiative (WHI), which showed negative effects, such as increased risk of cardiovascular disease and breast cancer. ${ }^{4,5}$ After this study, the prescription of hormone therapy dropped worldwide, ${ }^{3}$ including Brazil. Several non-hormonal drugs have been tested with significant reduction of hot flashes, ${ }^{6}$ but with significant side effects that prevented their use for longer periods of time. Thus, researchers have begun to review the WHI, and other studies, to better understand the risk of cardiovascular disease and its association with the use of hormone therapy.

After great reflection among societies studying climacteric, the concept of window of opportunity for hormonal therapy (HT) was created. It corresponds to the earliest time period to the start of HT. There is consensus on the introduction of HT up to 10 years after menopause, or until the age of 60 years. ${ }^{7-9}$ After this time, it is believed that endothelial dysfunction will be more advanced with established atheromatosis, preventing vasodilation determined by nitric oxide, and facilitating platelet aggregation. There would also be an increase in coagulation factors after the introduction of estrogen therapy associated with progestogen.

It seems that the window of opportunity may explain, in part, the concern for cardiovascular disease, but it does not explain the unease about breast cancer with the use of estroprogestative therapy. Perhaps one way to solve this is to better assess the breast risk of these patients, especially in terms of family or personal history, when there is ductal or lobular hyperplasia with atypia. In these cases, thorough thinking must be given to whether to introduce HT, or not. If necessary, employ lower doses in order to reduce menopausal symptoms and, whenever possible, use progestogens every three or four months to prevent endometrial proliferation and the increased risk of endometrial cancer. In women who have undergone hysterectomy, this concern does not exist because, in general, progestogen is not used. ${ }^{10}$

It should be noted that if the patient already has cardiovascular disease or any habit that increases cardiovascular risk, such as smoking, drinking and sedentary lifestyle, perhaps the window of opportunity has already been lost, because the endothelial dysfunction may be more advanced. There is much discussion about whether the adoption of healthy habits and elimination of addictions could be an alternative to mitigate the risks, allowing the use of hormonal therapy. ${ }^{11,12}$ Despite being an alternative to its use, prospective and well-controlled studies are insufficient to state that this therapeutic modality is without risk.

Thus, hormone therapy should not be recommended for all climacteric women, as not all women experience symptoms that interfere with their quality of life and others have contraindications that prevent their use. Estrogen therapy is still the best way to suppress hot flashes and increase urogenital tropism. Moreover, it assists in reducing the risk of osteoporotic fracture. Dose and duration of treatment should be individualized, though, and discussed in each medical consultation. Many women can benefit from estrogen therapy; not for the sake of being young forever, but to have good quality of life.

\section{References}

1. Galhardo CL, Soares JM Jr, Simões RS, Haidar MA, Rodrigues de Lima G, Baracat EC. Estrogen effects on the vaginal $\mathrm{pH}$, flora and cytology in late postmenopause after a long period without hormone therapy. Clin Exp Obstet Gynecol. 2006;33(2):85-9. 
2. Da Fonseca AM, Bagnoli VR, Souza MA, Azevedo RS, Couto Ede B Jr, Soares $\mathrm{JM} \mathrm{Jr}$, Baracat EC. Impact of age and body mass on the intensity of menopausal symptoms in 5968 Brazilian women. Gynecol Endocrinol. 2013;29(2):116-8.

3. Chlebowski RT, Anderson GL. Menopausal hormone therapy and breast cancer mortality: clinical implications. Ther Adv Drug Saf. 2015;6(2):45-56.

4. Houck JA. "What do these women want?": Feminist responses to Feminine Forever, 1963-1980. Bull Hist Med. 2003 Spring;77(1):103-32.

5. Lobo RA. Where are we 10 years after the Women's Health Initiative? J Clin Endocrinol Metab. 2013;98(5):1771-80.

6. Mintziori G, Lambrinoudaki I, Goulis DG, Ceausu I, Depypere H, Erel CT, et al. EMAS position statement: Non-hormonal management of menopausal vasomotor symptoms. Maturitas. 2015 (in press)

7. Sood R, Faubion SS, Kuhle CL, Thielen JM, Shuster LT. Prescribing menopausal hormone therapy: an evidence-based approach. Int J Womens Health. 2014;06:47 AM-57.
8. Wender COM, Pompei LM, Fernandes CE. Consenso Brasileiro de Terapêutica Hormonal da Menopausa - Associação Brasileira de Climatério (SOBRAC) - São Paulo: Leitura Médica, 2014.

9. Guidozzi F, Alperstein A, Bagratee JS, Dalmeyer P, Davey M, De Villiers TJ, et al. South African Menopause Society revised consensus position statement on menopausal hormone therapy, 2014. S Afr Med J. 2014; 104(8):537-43

10. Mirkin S, Archer DF, Taylor HS, Pickar JH, Komm BS. Differential effects of menopausal therapies on the endometrium. Menopause. 2014 21(8):899-908

11. Chatkin R, Chatkin JM, Spanemberg L, Casagrande D, Wagner M, Mottin C. Smoking is associated with more abdominal fat in morbidly obese patients PLoS One. 2015;10(5):e0126146.

12. Pines A. Sedentary women: sit less, live more! Climacteric. 2015 Apr 02:01 PM-3. 